\title{
Detailed balance method for ideal single-stage fluorescent collectors
}

\author{
T. Markvart ${ }^{\text {a) }}$ \\ School of Engineering Sciences, University of Southampton, Southampton SO17 1BJ, United Kingdom
}

(Received 1 June 2005; accepted 23 November 2005; published online 18 January 2006)

\begin{abstract}
It is shown that fluorescent collectors where radiation is confined with the use of selective reflectors can be modeled as converters of blackbody radiation. By decreasing the temperature and frequency of the radiation, the effective étendue of the emitted beam can be reduced substantially in the conversion process without violating the second law of thermodynamics. This type of collector can, in principle, achieve surprisingly high efficiencies: the output from a silicon solar cell operating with an ideal collector can exceed $90 \%$ of the output from a directly illuminated solar cell. (C) 2006 American Institute of Physics. [DOI: 10.1063/1.2160710]
\end{abstract}

Fluorescent concentrators/collectors have attracted interest for some time [see, for example, (Refs. 1 and 2)]. In common with other concentrating systems these devices aim to reduce the size of the solar cell, thereby decreasing the overall system cost. Unlike passive (geometric) concentrators, fluorescent collectors can utilize diffuse sunlight and need not therefore track the sun. ${ }^{3}$

A typical fluorescent collector absorbs incident light through the front face of a luminescent plate. The fluorescent light is trapped by total internal reflection (TIR) and, when used in photovoltaic applications, is guided onto a solar cell positioned at the edge of the plate. The absorbing/fluorescent medium may consist of dye modules, quantum dots, or nanocrystals. ${ }^{4}$ Indeed, other media can, in principle, be used, including semiconductors with a long minority-carrier lifetime.

This article considers a similar concept (originally suggested in Ref. 5) where fluorescent light is confined within the collector by a spectrally selective reflector ("hot mirror") which covers the top surface. An important additional feature not considered in Ref. 5 is perfect photon recycling within the collectors. We show that this arrangement produces inherently more efficient photon confinement than geometric trapping by TIR or by a hot mirror alone, and provides a natural framework for the discussion of an ideal single-stage collector. In effect, we apply the celebrated detailed balance method developed in Ref. 6 for solar cells to fluorescent collectors. More precisely, we follow the variant formulated by Ruppel and Würfel ${ }^{7}$ who introduced a nonzero chemical potential for radiation. We shall call such radiation quasiblackbody radiation.

Let us consider, quite generally, a fluorescent collector of arbitrary shape, consisting of a medium with refractive index $n$ which perfectly absorbs and emits all radiations with frequency $\nu>\nu_{g}$, in other words, a blackbody in this restricted frequency range. Blackbody radiation at temperature $T_{\text {in }}$ is incident from air or vacuum onto an area $A_{\text {ent }}$ of the collector. This entrance aperture is covered with a selective reflector which transmits only radiation with frequency $\nu>\nu_{\text {abs }}\left(>\nu_{g}\right)$; other radiation is reflected.

a)Electronic mail: tm3@soton.ac.uk
The incident radiation excites fluorescent medium inside the collector which then emits radiation through an exit aperture of area $A_{\text {exit }}$ in the full frequency range $\nu>\nu_{g}$. Some radiation with $\nu>\nu_{\text {abs }}$ will, of course, also escape through entrance aperture (Fig. 1). A solar cell with band gap $h \nu_{g}$ is attached to the exit aperture. Save for the entrance and exit apertures, all other surfaces of the collector are perfectly reflecting. We assume that the fluorescence takes place with a quantum yield of unity, and the photon flux is therefore conserved.

The complete absorption for $\nu>\nu_{g}$ by the collector medium implies that all the fluorescent light is reabsorbed, and is gradually brought into thermal equilibrium with the absorbing/fluorescent species in the collector before reaching the solar cell. This perfect reabsorption is analogous to photon recycling discussed in connection with radiative transitions in semiconductors ${ }^{8}$ and solar cells. ${ }^{9}$ The quasiequilibrium nature of the emitted photon flux indicates that the photon occupation numbers follow the Bose-Einstein distribution,

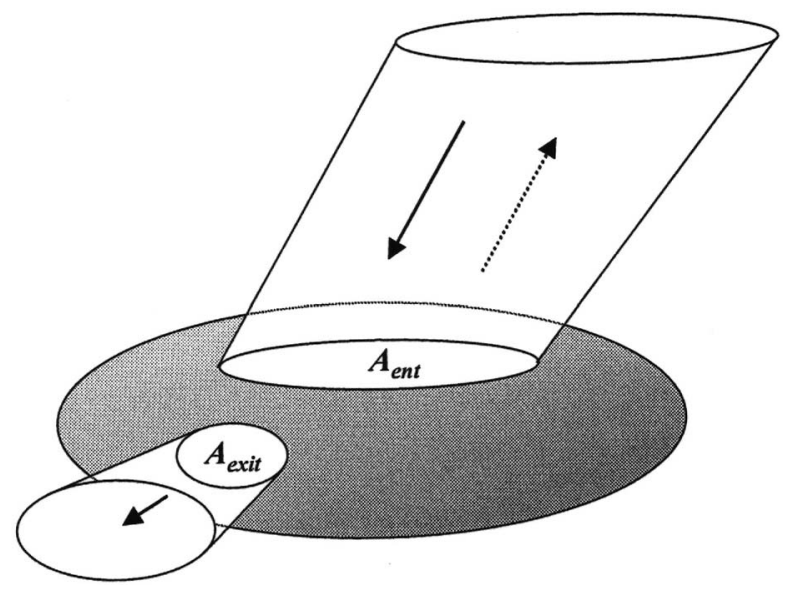

FIG. 1. A schematic representation of the fluorescent collector considered in this work. 


$$
\rho_{\nu}(T, \mu)=\frac{1}{e^{(h \nu-\mu) / k T_{0}}-1},
$$

with $T_{0}$ equal to the temperature of the collector. As discussed in Ref. 7, a nonzero chemical potential is enforced by the conservation of the photon flux.

The collector can therefore be described as a converter of blackbody radiation: a high-temperature beam in the frequency range $\nu>\nu_{\text {abs }}$ is transformed into a beam at a lower temperature in the frequency range $\nu>\nu_{g}$. This approach extends the pioneering theory of Yablonovich ${ }^{10}$ who considered a fluorescent collector with line absorption and fluorescent spectra.

The photon fluxes can be conveniently discussed in terms of étendue $\mathcal{E}$,

$$
\dot{N}=\mathcal{E} \int_{\nu_{0}}^{\infty} \frac{2 \nu^{2}}{c^{2}} \rho_{\nu}(T, \mu) d \nu
$$

where the frequency limit $\nu_{0}$ equals $\nu_{\text {abs }}$ or $\nu_{g}$ for radiation passing through the entrance or exit aperture, respectively. The étendue of the incident radiation is equal to $A_{\mathrm{ent}} \omega_{s}$ for a source of the incident beam (for example, the sun) which subtends an angle $\omega_{s}$. The étendue of the beam emitted through the entrance aperture is, similarly, $\mathcal{E}_{\text {ent }}=\pi A_{\text {ent }}$. Assuming that the solar cell material has a higher refractive index than the medium of the collector, the beam emitted through the exit aperture extends over the full hemisphere $(2 \pi)$, with an étendue $\mathcal{E}_{\text {exit }}=\pi n^{2} A_{\text {exit }}$. The efficiency of the collector is then given by $\eta=\dot{N}_{\text {exit }} /\left(\dot{N}_{\text {exit }}+\dot{N}_{\text {ent }}\right)$, where $\dot{N}_{\text {ent }}$ and $\dot{N}_{\text {exit }}$ are the total numbers of photons emitted through the entrance and exit apertures per unit time.

The formalism becomes particularly simple for lowintensity illumination such as sunlight where stimulated emission can be neglected and the photon flux resembles the ideal gas,

$$
\dot{N} \cong \mathcal{E}(T) e^{\left(\mu-h \nu_{0}\right) / k_{B} T_{0}}
$$

where

$$
\mathcal{E}(T)=\mathcal{E} \frac{2 \nu_{0}^{2} k_{B} T}{h c^{2}}\left[1+\varepsilon\left(\nu_{0}, T\right)\right],
$$

and $\varepsilon(\nu, T)=2\left(k_{B} T / h \nu\right)+2\left(k_{B} T / h \nu\right)^{2}$ is a small correction term. Since the chemical potential is the same for all emitted radiation, we readily obtain

$$
\eta=\frac{1}{1+\left[\mathcal{E}^{\mathrm{ent}}\left(T_{0}\right) / \mathcal{E}^{\mathrm{exit}}\left(T_{0}\right)\right] e^{-h \Delta \nu / k_{B} T_{0}}},
$$

where $\Delta \nu=\nu_{\mathrm{abs}}-\nu_{g}$.

The chemical potential can be determined by equating the incident flux to the total emitted flux $\dot{N}_{\text {exit }}+\dot{N}_{\text {ent }}$,

$$
\begin{aligned}
\mu= & h \nu_{\mathrm{abs}}\left(1-\frac{T_{0}}{T_{\mathrm{in}}}\right)+k_{B} T_{0} \ln \left(\frac{\omega_{s}}{\pi} \frac{T_{\text {in }}}{T_{0}}\right) \\
& +k_{B} T_{0} \ln \left[\frac{1+\varepsilon\left(\nu_{\mathrm{abs}}, T_{0}\right)}{1+\varepsilon\left(\nu_{g}, T_{0}\right)}\right]+k_{B} T_{0} \ln (1-\eta) .
\end{aligned}
$$

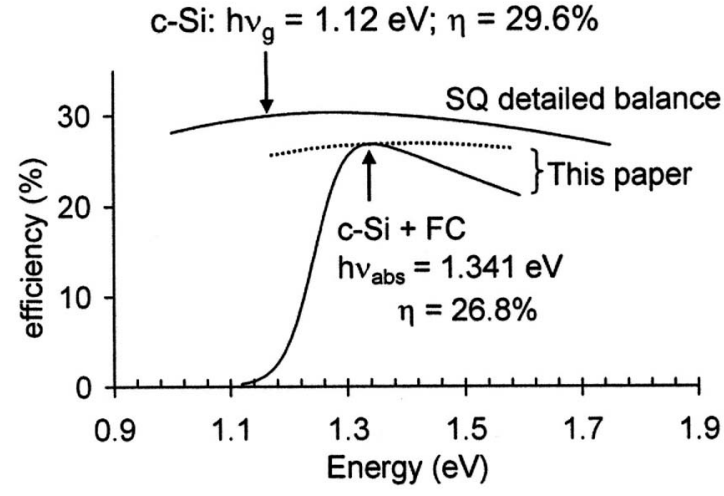

FIG. 2. The conversion efficiency of an ideal solar cell given by the Shockley-Queisser (SQ) detailed balance limit as a function of the band gap $h \nu_{g}$, and of a silicon solar cell $\left(h \nu_{g}=1.12 \mathrm{eV}\right)$ with a fluorescent collector as a function of the energy $h \nu_{\text {abs }}$. The full line corresponds to a fixed $\mathcal{E}_{\text {ent }} / \mathcal{E}_{\text {exit }}=100$; the dotted line corresponds to an optimum ratio $\mathcal{E}_{\text {ent }} / \mathcal{E}_{\text {exit }}$ for each $\nu_{\text {abs }} . T_{\text {in }}=6000 \mathrm{~K}$ and $T_{0}=300 \mathrm{~K}$.

Equation (6) assumes that the chemical potential of the incident radiation is zero. This corresponds to, for example, the direct (beam) solar radiation but can easily be extended to diffuse radiation, which has a larger étendue and a negative chemical potential.

The first three terms in (6) represent an approximation for the open circuit voltage of a solar cell made from semiconductor with band gap $h \nu_{\text {abs }}$. The first two terms have been obtained in Ref. 7 but it is the third term which gives a usable formula: for example, for radiation with one-sun intensity and a solar cell with band gap in excess of approximately $1 \mathrm{eV}$, the accuracy of (6) is better than $1 \mathrm{meV}$. The last term in (6) gives a reduction in $\mu$ on account of the collector.

Formulas (5) and (6) can be used to calculate accurately the total power produced by the combined system of a fluorescent collector and an ideal single-junction solar cell which captures all light emitted through the exit aperture. Such cell will produce voltage $\mu / q$, where $\mu$ is given by Eq. (6), and current which can easily be obtained from the incident photon flux [obtained from the full Eq. (2) to maintain good accuracy] and the photon flux conversion efficiency $\eta$ given by (5). The energy conversion efficiency of such a combined system is shown in Fig. 2.

Thus, we have obtained the efficiency of an ideal singlestage fluorescent collector which emits quasiblackbody radiation, and where light is confined by means of a spectrally selective mirror placed on the entrance aperture of the collector. The use of the detailed balance formalism makes it possible to calculate the efficiency of the collector in rather general terms. A collector with ratio $\mathcal{E}_{\text {ent }} / \mathcal{E}_{\text {exit }}=100$ (realized, for example, by a medium with $n=1.7$ and an area concentration ratio of 289) gives a power efficiency limit of $26.8 \%$, some $90 \%$ of the maximum efficiency of an ideal silicon solar cell. This high value exceeds substantially the overall conversion efficiencies of less than $10 \%$ usually predicted for the conventional TIR or hot mirror devices. ${ }^{5}$ 
It is a pleasure to acknowledge many stimulating discussions with my colleagues Peter Lansdsberg, Lefteris Danos, Robert Greef, and Pat Kittidachachan on the subject of this paper.

${ }^{1}$ W. H. Weber and J. Lambe, Appl. Opt. 15, 2299 (1976).

${ }^{2}$ A. Goetzberger and W. Greubel, Appl. Phys. 14, 123 (1977).

${ }^{3}$ See, for example, G. Smestad, H. Ries, R. Winston, and E. Yablonovich, Sol. Energy Mater. 21, 95 (1990).
${ }^{4}$ K. Barnham, J. L. Marques, J. Hansard, and P. O’Brien, Appl. Phys. Lett. 76, 1197 (2000)

${ }^{5}$ B. S. Richards, A. Shalav, and R. P. Corkish, Proceedings of the 19th European Photovoltaic Solar Energy Conference, 2004, p. 113.

${ }^{6}$ W. Shockley and H. J. Queisser, J. Appl. Phys. 32, 510 (1961).

${ }^{7}$ W. Ruppel and P. Würfel, IEEE Trans. Electron Devices ED-27, 877 (1980).

${ }^{8}$ O. von Roos, J. Appl. Phys. 54, 1390 (1983).

${ }^{9}$ J. E. Parrott, Sol. Energy Mater. Sol. Cells 30, 221 (1993).

${ }^{10}$ E. Yablonovich, J. Opt. Soc. Am. 70, 1362 (1980). 